\title{
New conceptual microfluidics technology: light manipulation of liquid slugs in liquid crystal polymer microactuators
}

\author{
Zhichao Dong and Lei Jiang*
}

Microsystems that manipulate small amounts of fluids to transport in a pre-defined direction and to perform reactions or analyses are quite important in both laboratory investigations and industry applications [1], due to their close relevance to people's daily life and commercial run. Natural creatures, after centuries' evolution, have realized the importance of structure and wettability designs in achieving liquid directional transportation-biological structural materials frequently harness "the Laplace effect" to transport liquid for survival. To achieve "Laplace pressure" difference, the surface gradient plays a vital role in achieving unbalanced surface tension at either side of the liquid dropped on solid surfaces. Cone is a typical gradient structure for liquid motion, and biological examples include, spider silk, which takes advantage of surface gradient between spindle-knots and joints to transport water droplets directionally from the joints to spindle-knots [2], and drought-tolerant cactus, exploiting shape gradient along a single cactus spine to produce a directional liquid transport system. According to the classical Chinese philosophy, two entirely opposite things could be cooperative [3]. Compared with the conical structures, cavity and beak structures have been utilized by the pitcher plant [4] and shorebirds [5] to achieve liquid directional transportations, respectively. As shown in Fig. 1b, a wetting liquid droplet confined in a conical capillary could self-propel toward the narrower end, owing to the axial force arising from different curvature pressures across its end caps. Inspired by these special surfaces, various methods have been developed to prepare surfaces with similar directional liquid transport abilities and designed for practical applications, such as liquid transportation, liquid mixture, water harvest, liquid-liquid separation, and bubble collection [6,7].

However, because of the length limit of these cone or beak structures, liquid cannot transport for a long distance. Injecting energy, such as light [8], vibrations [5] or heat, into the system is thus an alternative method for liquid motion. Significantly, light radiation, light modulation of electrical actuation (optoelectrowetting and photocontrol of electroosmotic flow) or light-induced capillary force [8], is a facile method to induce liquid motion, which could provide contactless, spatial, temporal and precise control. Though effective in these cases, we need to notice that several drawbacks still exist, where the capillary force arising from wettability gradient is too small to overcome the effect of contact line pinning. The motion is thus limited to specific liquids with a relatively short distance, simple linear trajectories, and low speed. On the other hand, light-induced "Marangoni effect" requires either local heating or adding photosensitive surfactants into liquids, which is undesirable for biomedical application and undoubtedly produces pollution. The design of energy injection needs to be adjusted. As a hypothesis, if it is possible to build tubular micro-actuators whose geometry can be dynamically adjusted by light to transverse between column and cone, a simple and straightforward method to manipulate long distance and high speed liquid transportation will be achieved.

Recently, Professor Yanlei Yu at Fudan University and co-workers proposed a new strategy to manipulate liquids by photo-induced asymmetric deformation of tubular microactuators [1]. They ingeniously designed a linear liquid crystal polymer (LLCP) which was inspired from the lamel-

Key Laboratory of Bio-inspired Smart Interface Sciences, Technical Institute of Physics and Chemistry, Chinese Academy of Sciences, Beijing 100190, China

*Email: jianglei@iccas.ac.cn 


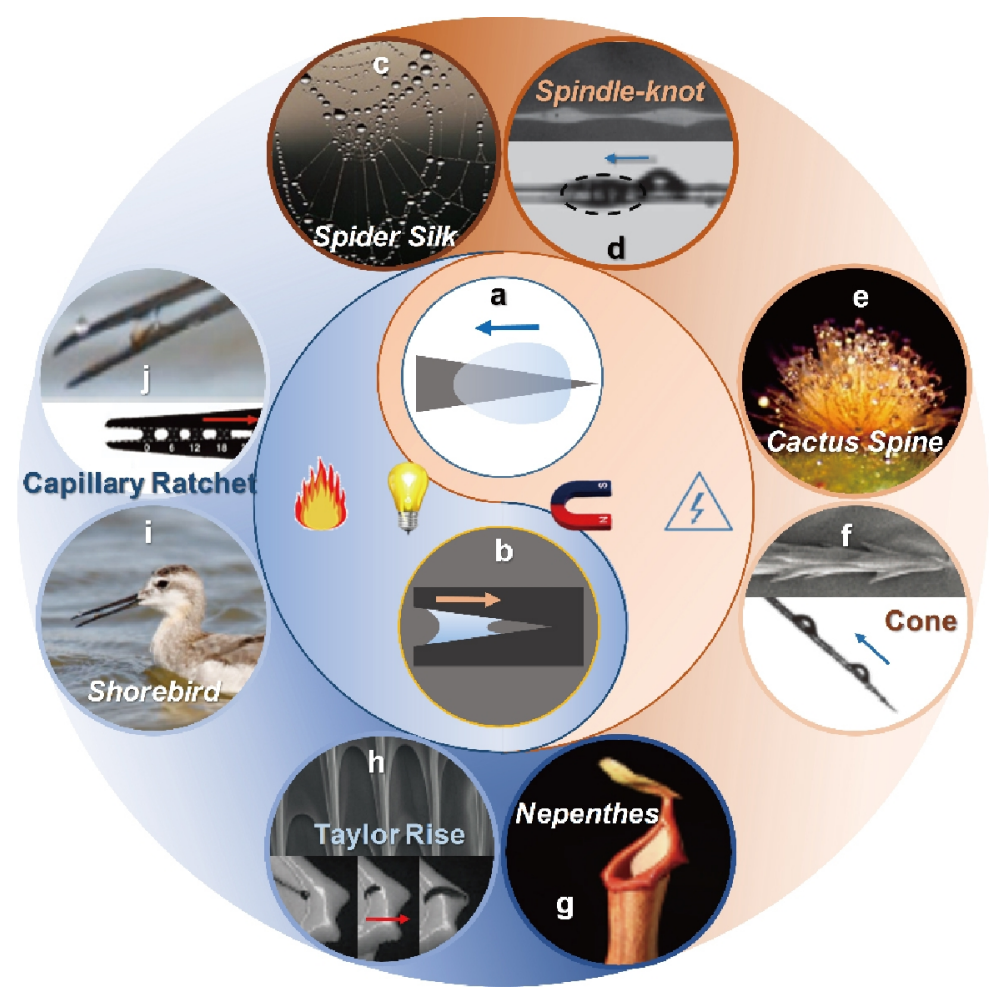

Figure 1 Schematic illustration of "the Laplace pressure" induced liquid directional motion. (a, b) Cone and beak are two typical gradient structures for liquid motion. Due to the curvature differences of the liquid film on the cone or beak surface, liquid drop can be forced by "the Laplace pressure" to move in a single direction. Natural creatures can harness this kind of surface gradient to transport liquid for survival. (c, d) Spider silk takes advantage of surface gradient between spindle-knots and joints to transport water droplets directionally from joints to spindle-knots. (e, f) Drought-tolerant cactus exploits shape gradient along a single cactus spine to produce a directional liquid transport system. (g, h) Pitcher plant utilizing its arrayed micro-cavities to achieve liquid directional transportations. (i, j) Shorebird succeeds in raising the drops into their mouths by opening and closing their beaks successively. As a hypothesis, integrating surface gradient and injecting energy, such as light, electric field, heat, or magnetic field, along a surface would produce a long-distance and high-speed liquid motion.

lar structure of artery wall to ensure enough mechanical robustness without chemical crosslinks. Through a facile solution processing, structurally defined and robust tubular microactuators can be fabricated, which provides a straightforward and versatile solution to precisely handle liquids over a long distance with desirable velocity and controllable direction. It is noteworthy that state-of-the-art tubular microactuators take advantage of an entirely new principle to propel liquids by capillary force arising from photo-induced asymmetric deformation (Fig. 2a and $b$ ), which relies on neither wettability gradient nor "Marangoni effect". This asymmetric deformation arises from 470-nm light-induced reorientation of azobenzene mesogens, introducing a decrease of thickness of the wall of tubular microactuator and an elongation of perimeter of the tubular microactuator. Then it causes the increase of cross-sectional area of the tubular microactuator. The higher the light intensity is, the larger the cross-sectional area becomes. Thus, the tubular microactuator deforms to an asymmetric cone-like geometry which generates adjustable capillary force to propel liquids towards the light attenuated direction (Fig. 2a). The tubular microactuators are applicable to propel not only simple liquids spanning a broad range of polarity, but also complex fluids widely used in biomedical and chemical engineering, thus having considerable potential applications in microsystems technology and architecture as micro-pumps without any aid of additional components.

Liquid handling is a most common and extremely important process in the fields of biological and chemical analysis and micro engineering. Achieving liquid motion driven by light in a S-shaped or helical trajectories is important for liquid handling but quite a hard work, and this kind of motion has never been realized in any closed microchannel until now. Yu and co-workers have achieved light-driven motion through tubular microactuators. To promote mechanical robustness of liquid crystal polymer, ring-opening metathesis polymerization (ROMP) was utilized. ROMP is 

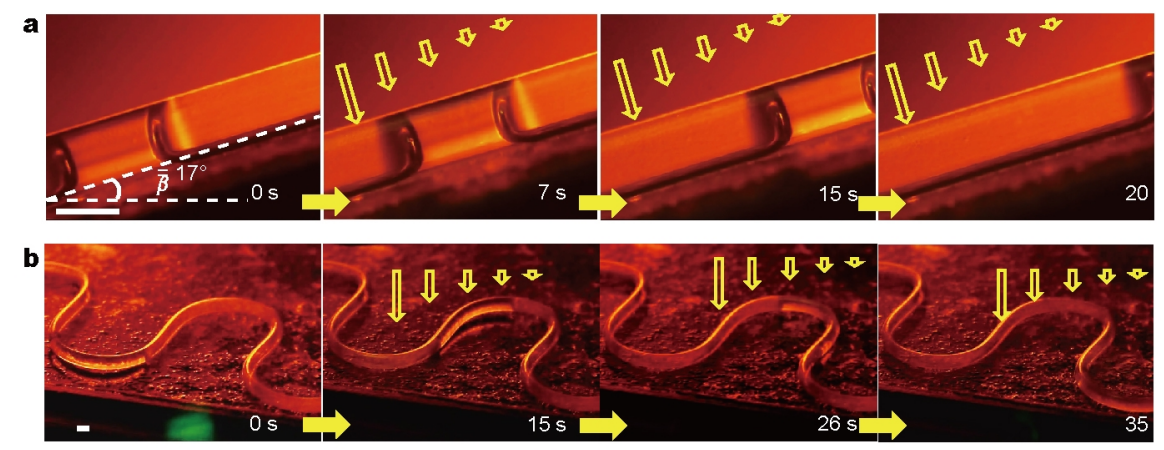

c
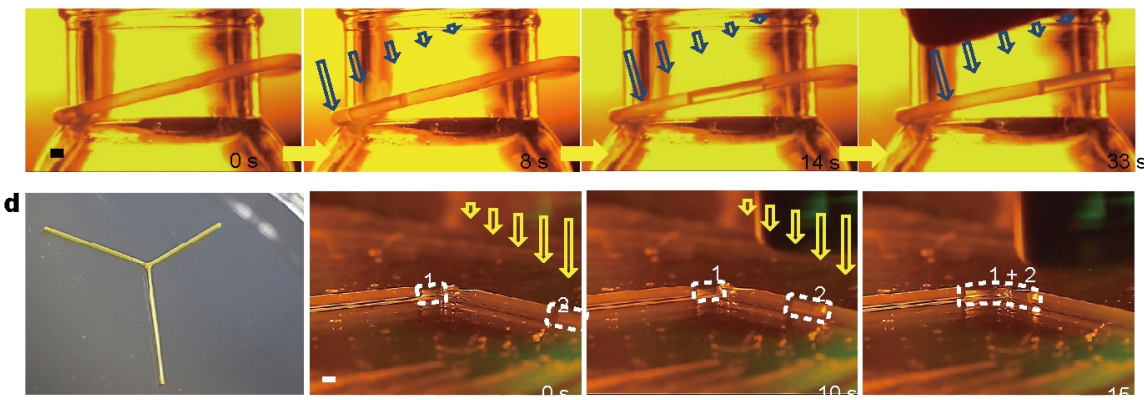

Figure 2 (a) Schematics showing the motion of a wetting liquid slug confined in a tubular microactuator (TMA) driven by photodeformation; (b) lateral photographs of light-induced motion of a silicone oil slug in the TMA. Upon irradiation of attenuated 470-nm light, the silicone oil slug is self-propelled towards the light attenuated direction and the moving direction of the slug is controllable by varying the attenuated direction; (c) lateral photographs showing light-driven motion of a silicone oil slug in the serpentine TMA; (d) optical image of the Y-shaped TMA and lateral photographs showing light-driven liquid fusion of two silicone oil slugs $\mathbf{1}$ and $\mathbf{2}$ at the junction of the Y-shaped TMA. Adapted with permission from Ref. [1], Copyright 2016, Macmillan Publishers Limited, part of Springer Nature.

a living polymerization process that allows synthesis of a high-molecular-weight polyolefin with narrow polydispersity. The number-average molecular mass of the liquid crystal polymer used in their work reaches $3.6 \times 10^{5}$ $\mathrm{g} \mathrm{mol}^{-1}$, which is at least one order magnitude larger than that of traditional azobenzene polymers based on acrylic backbone. Tensile tests in their article show that the LLCP fiber owns moderate elastic modulus ( $96 \pm 19 \mathrm{MPa})$, high toughness $\left(319 \pm 41 \mathrm{MJ} \mathrm{m}^{-3}\right)$, high strength $(\sim 20 \mathrm{MPa})$ and large elongation at break (2089 $\pm 275 \%)$, which ensures the repeatable photo-deformation. As a significant breakthrough in the fabrication of bio-inspired microactuator, commented by the anonymous reviewers ("this manuscript represents a very nice piece of work with real openings" and "the method superior to all existing technologies...not only for the new concept it reports (light-driven transport of liquids through photodeformation), but also for its broad applicability in terms of liquid types and the different light-triggered operations it allows"), this innovative strategy is applicable to a wide range of applications including liquid mixing, coalescence operation, and even capture and convey of the microsphere in microscale, which would sharply simplify microfluidic devices (Fig. 2d). These photo deformable tubular microactuators are considered as excellent candidates for applications in the fields of micro-reactors, laboratory-on-a-chip contexts and micro-optomechanical systems.

Received 21 September 2016; accepted 22 September 2016; published online 19 October 2016

1 Lv JA, Liu Y, Wei J, et al. Photocontrol of fluid slugs in liquid crystal polymer microactuators. Nature, 2016, 537: 179-184

2 Zheng Y, Bai H, Huang Z, et al. Directional water collection on wetted spider silk. Nature, 2010, 463: 640-643

3 Liu M, Jiang L. Dialectics of nature in materials science: binary cooperative complementary materials. Sci China Mater, 2016, 59: 239-246

4 Chen H, Zhang P, Zhang L, et al. Continuous directional water transport on the peristome surface of Nepenthes alata. Nature, 2016, 532: 85-89

5 Prakash M, Quere D, Bush JWM. Surface tension transport of prey by feeding shorebirds: the capillary ratchet. Science, 2008, 320: 931-934

6 Yu C, Cao M, Dong Z, et al. Spontaneous and directional transportation of gas bubbles on superhydrophobic cones. Adv Funct Mater, 2016, 26: 3236-3243

7 Tian D, Zhang N, Zheng X, et al. Fast responsive and controllable liquid transport on a magnetic fluid/nanoarray composite interface. ACS Nano, 2016, 10: 6220-6226

8 Ichimura K, Oh SK, Nakagawa M. Light-driven motion of liquids on a photoresponsive surface. Science, 2000, 288: 1624-1626 